\title{
Conditional Dynamics of Optomechanical Two-Tone Backaction-Evading Measurements
}

\author{
Matteo Brunelli, ${ }^{1}$ Daniel Malz, ${ }^{2}$ and Andreas Nunnenkamp ${ }^{1}$ \\ ${ }^{1}$ Cavendish Laboratory, University of Cambridge, Cambridge CB3 OHE, United Kingdom \\ ${ }^{2}$ Max-Planck-Institut für Quantenoptik, Hans-Kopfermann-Strasse 1, D-85748 Garching, Germany
}

(Received 15 March 2019; published 29 August 2019)

\begin{abstract}
Backaction-evading measurements of mechanical motion can achieve precision below the zero-point uncertainty and quantum squeezing, which makes them a resource for quantum metrology and quantum information processing. We provide an exact expression for the conditional state of an optomechanical system in a two-tone backaction-evading measurement beyond the standard adiabatic approximation and perform extensive numerical simulations to go beyond the usual rotating-wave approximation. We predict the simultaneous presence of conditional mechanical squeezing, intracavity squeezing, and optomechanical entanglement. We further apply an analogous analysis to the multimode optomechanical system of two mechanical and one cavity mode and find conditional mechanical Einstein-Podolski-Rosen entanglement and genuinely tripartite optomechanical entanglement. Our analysis is of direct relevance for ultrasensitive measurements and measurement-based control in high-cooperativity optomechanical sensors operating beyond the adiabatic limit.
\end{abstract}

DOI: 10.1103/PhysRevLett.123.093602

Introduction.-The standard quantum limit (SQL) is the precision limit that arises from the fundamental trade-off between the information extractable from a measurement and the associated backaction when continuously monitoring the mechanical motion [1,2]. Backaction-evading (BAE) measurements bypass this limit by restricting the measurement to a single quadrature of motion [3-5]. One way to implement this is to parametrically couple the mechanical motion to a cavity driven on both mechanical sidebands [3,6]. BAE measurements have been demonstrated in optomechanics, with sensitivities approaching the SQL [7-9], and exploited to generate spin squeezing in light-controlled atomic ensembles [10]. They have also been extended to collective observables of two modes [11-17].

Recent experimental advances have allowed us to access the conditional dynamics and real-time feedback of weakly monitored optomechanical systems at the quantum limit [18-21]. In BAE measurements, continuous monitoring would enable uncertainties below the SQL and the generation of conditional mechanical squeezing [22-24], which is a valuable resource in quantum metrology $[25,26]$ and continuous-variable quantum information [27-29]. Surprisingly, the current literature only considers an approximate description of this process, based on the intracavity field adiabatically following the mechanical motion [30]. With state-of-the-art cavity optomechanics experiments operating in the backaction-dominated regime [31], this description has become inadequate.

In this Letter, we present an exact treatment of the conditional dynamics of BAE measurements beyond adiabatic elimination and valid for initial Gaussian states. We

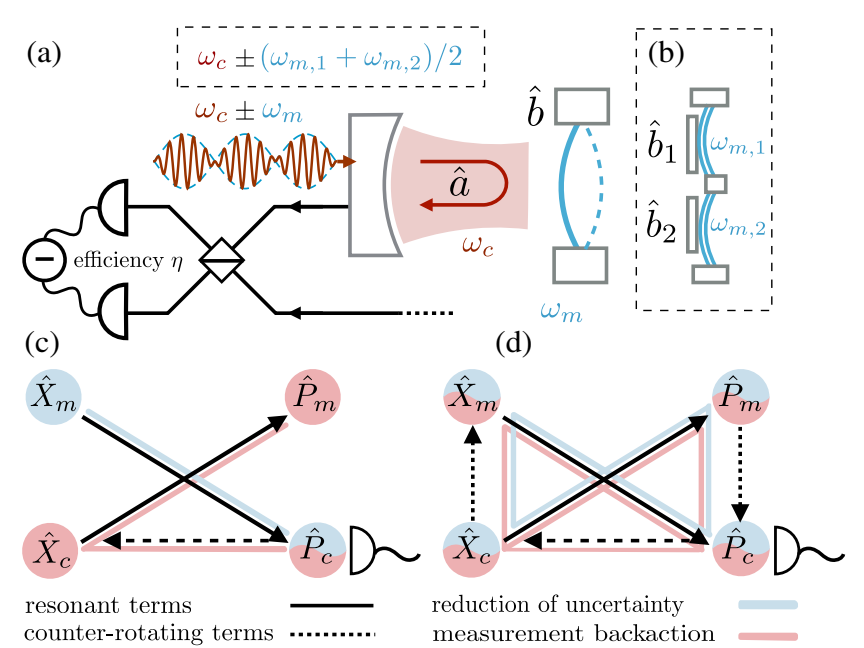

FIG. 1. (a) Backaction-evading (BAE) measurement of a single mechanical quadrature. An optomechanical cavity $(\hat{a})$ is driven on the lower and upper mechanical $(\hat{b})$ sideband and is continuously monitored via the output homodyne current. (b) If two mechanical modes $\hat{b}_{1}$ and $\hat{b}_{2}$ are considered instead (dashed boxes), a two-mode BAE measurement is realized. (c) Monitoring of the output field both introduces backaction and allows us to extract information. Arrows originate from the source terms in the equations of motion derived from Eq. (2). Within RWA, backaction is confined to $\hat{P}_{m}$ and reduction of uncertainty to $\hat{X}_{m}$. (d) Counter-rotating terms open new channels, which corrupt the BAE regime and reduce squeezing in $\hat{X}_{m}$ but, at the same time, enable joint reduction of uncertainty of other variables, e.g., $\hat{X}_{c}$ and $\hat{P}_{m}$; thus, correlations are enhanced and robust entanglement can be generated subject to measurement. 
predict the existence of an optimal value of mechanical squeezing (in terms of the system's parameters). Then, we numerically go beyond the rotating-wave approximation (RWA) and describe the quantum features induced by the measurement on the whole optomechanical system (conditional intracavity squeezing and optomechanical entanglement) which are entirely missed by taking the adiabatic approximation.

Finally, we extend our analysis to two mechanical modes coupled to a common cavity field. We show both conditional generation of mechanical Einstein-Podolski-Rosen (EPR) as well as genuine tripartite optomechanical entanglement. Our study provides a substantial improvement in the description of weakly monitored optomechanical systems (as well as parametrically coupled superconducting circuits [32-34]) and opens novel avenues for ultrasensitive measurements and measurement-based quantum control of mechanical motion.

Optomechanical conditional dynamics.-We consider an optomechanical system where a mechanical oscillator of frequency $\omega_{m}$ modulates the frequency of a cavity mode $\omega_{c}$ [35]. The Hamiltonian is given by $(\hbar=1)$

$$
\begin{aligned}
\hat{H}= & \omega_{c} \hat{a}^{\dagger} \hat{a}+\omega_{m} \hat{b}^{\dagger} \hat{b}-g_{0} \hat{a}^{\dagger} \hat{a}\left(\hat{b}+\hat{b}^{\dagger}\right) \\
& +\mathcal{E}(t) \hat{a}^{\dagger}+\mathcal{E}^{*}(t) \hat{a},
\end{aligned}
$$

where $\hat{a}(\hat{b})$ describes the cavity (mechanical) mode, $g_{0}$ is the single-photon coupling strength, and the cavity is driven on both mechanical sidebands $\omega_{c} \pm \omega_{m}$ with the same strength, i.e., $\mathcal{E}(t)=2|\mathcal{E}| e^{-i \omega_{c} t} \cos \omega_{m} t$. After linearization and moving to an interaction picture with respect to the free mechanical and cavity evolution, we obtain

$$
\hat{H}_{I}(t)=-g \hat{X}_{c}\left[\hat{X}_{m}\left(1+\cos 2 \omega_{m} t\right)+\hat{P}_{m} \sin 2 \omega_{m} t\right],
$$

with coupling strength $g \equiv g_{0}|\mathcal{E}| / \sqrt{\omega_{m}^{2}+\kappa^{2} / 4}$, cavity decay rate $\kappa$, and dimensionless quadratures $\hat{X}_{c}=$ $\left(\hat{a}+\hat{a}^{\dagger}\right) / \sqrt{2}, \hat{X}_{m}=\left(\hat{b}+\hat{b}^{\dagger}\right) / \sqrt{2}$, and $\hat{P}_{m}=i\left(\hat{b}^{\dagger}-\hat{b}\right) / \sqrt{2}$. Equation (2) has a time-independent part, $\hat{H}_{\mathrm{QND}}=$ $-g \hat{X}_{c} \hat{X}_{m}$, and an oscillating part $\hat{H}_{\mathrm{CR}}(t)$. In the goodcavity limit $\kappa \ll \omega_{m}$, the latter can be neglected and the interaction is manifestly quantum non-demolition (QND) [6].

We also include interactions with the photonic and the mechanical environment [36]. Both environments consist of a collection of uncorrelated modes that interact with the system at time $t$ and are, otherwise, uncoupled; this assumption both gives rise to a Markovian environment and provides a monitoring channel. After interacting with the system, the photonic modes of the environment undergo a homodyne measurement of the phase quadrature $\hat{P}_{c}$ [23] [see Fig. 1(a)].

Given the (bi)linear nature of both the interaction and the measurement and given a Gaussian initial state, the state of the optomechanical system $\hat{\varrho}$ is exhaustively described in terms of the mean vector $\overline{\mathrm{x}}=\operatorname{Tr}[\hat{\varrho} \hat{\mathrm{x}}]$ and covariance matrix $(\mathrm{CM}) \sigma=\frac{1}{2} \operatorname{Tr}\left[\hat{\varrho}\left\{\hat{\mathrm{x}}-\overline{\mathrm{x}},(\hat{\mathrm{x}}-\overline{\mathrm{x}})^{T}\right\}\right]$, where we set $\hat{\mathrm{x}}=\left(\hat{X}_{c}, \hat{P}_{c}, \hat{X}_{m}, \hat{P}_{m}\right)^{T}$ [27]. The conditional evolution of the continuously monitored system is then described by the following set of equations [37,38]:

$$
\begin{gathered}
d \overline{\mathrm{x}}=A \overline{\mathrm{x}} d t-(\sigma B-N) d W_{t}, \\
\dot{\sigma}=A \sigma+\sigma A^{T}+D-(\sigma B-N)(\sigma B-N)^{T},
\end{gathered}
$$

where $A=A(t)$ is the drift matrix, $D$ the diffusion matrix, $B$ and $N$ account for the reduction of uncertainty and added noise due to the measurement; $W_{t}$ is a vector of independent Wiener processes $\left(d W_{j} d W_{k}=\delta_{j k} d t\right)$, see Supplemental Material (SM) [36]. Notice that the stochastic evolution, consequence of the measurement-induced disturbance, is confined to the first moments. Therefore, at any time, the conditional state is represented by a Gaussian state whose CM evolves deterministically according to Eq. (4). This will represent the main tool of our analysis.

Mechanical squeezing beyond adiabatic approximation.-We start by studying the conditional dynamics of a two-tone BAE measurement within the RWA, namely when Eq. (2) reduces to the perfect QND interaction $\hat{H}_{\mathrm{QND}}=-g \hat{X}_{c} \hat{X}_{m}$. The steady-state conditional CM (4) can be obtained analytically (cf. SM [36]). Here, we focus on the variances of the two mechanical quadratures

$$
\begin{gathered}
\sigma_{X_{m}}^{2}=\frac{\sqrt{\gamma^{2}+\kappa^{2}+2 \zeta}}{16 g^{2} \eta \kappa}\left(\zeta+\gamma^{2}-\gamma \sqrt{\gamma^{2}+\kappa^{2}+2 \zeta}\right), \\
\sigma_{P_{m}}^{2}=\bar{n}+\frac{1}{2}+\frac{2 g^{2}}{\gamma(\gamma+\kappa)},
\end{gathered}
$$

where $\zeta=\sqrt{\gamma \kappa\left[16 g^{2} \eta(1+2 \bar{n})+\gamma \kappa\right]}, \bar{n}$ is the thermal occupancy of the mechanical bath, and $0 \leq \eta \leq 1$ is the quantum efficiency of the measurement. These exact expressions are the first central result of our work.

We note that, for $\eta \rightarrow 0$, no measurement is recorded and Eq. (5) reduces to the unconditional variance $\sigma_{X_{m}}^{2} \rightarrow \bar{n}+\frac{1}{2}$, which is consistent with the fact that $\hat{X}_{m}$ is a conserved quantity. Physically, the presence of a monitoring channel introduces disturbance, which directly affects the conjugate quadrature $\left(\hat{X}_{c}\right)$ and, via the optomechanical coupling, leads to increased fluctuations in $\hat{P}_{m}$ (backaction heating) [cf. last term in Eq. (6) and Fig. 1(c)]. On the other hand, when the measurement is recorded $(\eta>0)$, information about the mechanical state is indirectly acquired, which reduces the uncertainty (variance) as shown by Eq. (5), eventually resulting in mechanical squeezing $\sigma_{X_{m}}^{2}<\frac{1}{2}$.

We show the degree of mechanical squeezing [expressed in $-10 \log _{10}\left(2 \sigma_{X_{m}}^{2}\right)$ Decibel (dB)] in Fig. 2, as a function of the sideband parameter $\kappa / \omega_{m}$. An optimal value of squeezing emerges for intermediate $\kappa / \omega_{m}$ as a result of the 


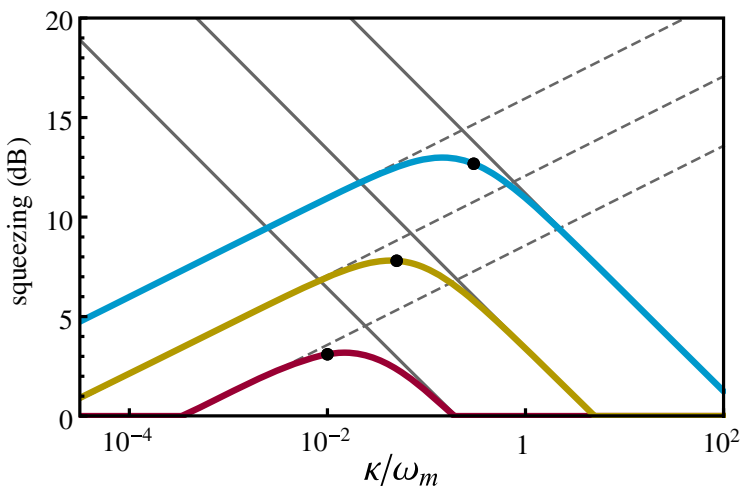

FIG. 2. Mechanical squeezing (in $\mathrm{dB}$ ) for $g=0.01 \omega_{m}$ (red), $g=0.05 \omega_{m}$ (yellow), and $g=0.3 \omega_{m}$ (cyan) as predicted by Eq. (5). Other parameters are $\gamma=10^{-4} \omega_{m}, \bar{n}=10, \eta=1$. Solid black lines represent the adiabatic solution $\sigma_{X_{m}}^{2}$ ad while dashed lines represent that of a slow cavity $\sigma_{X_{m}}^{2}$,slow. For each curve, the part to the left of the black dot $(g=\kappa)$ is in the strong-coupling regime.

competition between increasing the transfer of mechanical information to the light field and increasing the number of measured photons, which would favor, respectively, a slower and a faster cavity. For a fast cavity $\kappa \gg \omega_{m}$, we retrieve the adiabatic result $\sigma_{X_{m}}^{2} \approx \sigma_{X_{m} \text {,ad }}^{2}=$ $[\sqrt{1+4 \eta \mathcal{C}(1+2 \bar{n})}-1] / 4 \eta \mathcal{C}$ where we introduced the cooperativity $\mathcal{C}=4 g^{2} / \kappa \gamma$. This expression can also be obtained by adiabatically eliminating the cavity mode and considering the resulting effective measurement of $\hat{X}_{m}$ [39]; this is the standard approach for describing the conditional evolution of weakly monitored systems $[6,13,22,24,30,31,40]$. In the adiabatic regime, decreasing $\kappa$ leads to a larger cooperativity (and hence, to a larger effective measurement rate [6]) and determines a steady increase of squeezing. However, as our solution shows, when this rate becomes smaller than the rate at which mechanical information is imprinted onto the light field $(g)$, this description becomes inaccurate. For example, for $g=\kappa=10^{-2} \omega_{m}, \sigma_{X_{m} \text {,ad }}^{2}$ overestimates the actual amount of squeezing by approximately a factor of 2 (cf. Fig. 2). For a slow cavity $\kappa \ll \omega_{m}$, on the other hand, increasing $\kappa$ increases the measurement rate (more photons reaching the detector), which in turn reduces the variance $\sigma_{X_{m}}^{2}$. We can express Eq. (5) in terms of $\mathcal{C}$ and keep only the leading term in the expansion $\mathcal{C} \gg 1$, which yields $\sigma_{X_{m} \text {,slow }}^{2}=\left((1+2 \bar{n})^{3 / 4} /(\mathcal{C} \eta)^{1 / 4}\right) \sqrt{\gamma / \kappa}$ shown as dashed lines in Fig. 2. Once again, this description loses accuracy when the cavity loss becomes comparable to the coherent term. Our exact solution (5) interpolates between these two limits and describes a trade-off between two different measurement regimes.

A more accurate condition for optimal squeezing is obtained from the intersection of the two straight lines in Fig. 2

$$
\kappa_{\mathrm{opt}}=4 g^{2 / 3}[\eta \gamma(1+2 \bar{n})]^{1 / 3} .
$$

This gives the optimal value of the sideband parameter, which both depends on the rate at which information is transferred to the cavity and on the thermal decoherence rate.

Effects of counter-rotating terms.-Now, we explore the effect of the counter-rotating (CR) terms appearing in Eq. (2). As the drift matrix is explicitly time-dependent, we numerically integrate the equations of motion (4) and consider the long-time limit, when the system settles in a time-periodic steady state. The inclusion of the CR terms enables measurement backaction to reach $\hat{X}_{m}$ [see Fig. 1(d)] and, therefore, perturb the ideal QND regime. The consequent reduction of mechanical squeezing can be seen in Fig. 3(a). However, such a reduction is accompanied by the emergence of two novel features: (i) the
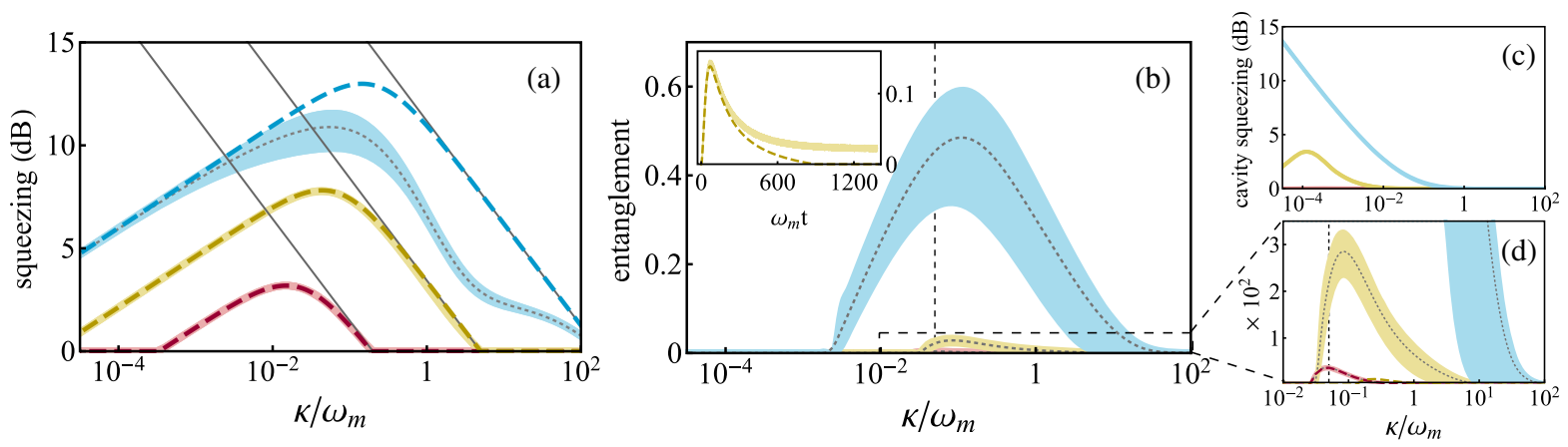

FIG. 3. (a) Mechanical squeezing (in dB) assuming the RWA [Eq. (5)] (dashed dark curves) and beyond the RWA (lighter shaded areas). The curves are for $g=0.01 \omega_{m}$ (red), $g=0.05 \omega_{m}$ (yellow), and $g=0.3 \omega_{m}$ (cyan); the dotted curve shows the mean squeezing (averaged over one mechanical period) and the shaded area extends between the minimum and maximum value of squeezing. Solid black lines represent the adiabatic solution $\sigma_{X_{m}}^{2}$,ad (b) Conditional entanglement (measured by the logarithmic negativity) for the same couplings as (a); the vertical dashed line corresponds to $\kappa=0.05 \omega_{m}$, and in the inset, we show the temporal evolution of entanglement along this cut for the case $g=0.05 \omega_{m}$. (c) Conditional cavity squeezing for the same couplings as (a). (d) Zoom-in of panel (b). In all panels, other parameters are: $\gamma=10^{-4} \omega_{m}, \bar{n}=10, \eta=1$. 
stabilization of optomechanical entanglement to considerably larger values [panels (b), (d)] and (ii) the appearance of squeezing in the cavity quadrature $\hat{X}_{c}$ [panel (c)]. In particular, the presence of CR terms can have a dramatic effect on entanglement, which survives in the steady state, as opposed to the typical entanglement "sudden death" predicted by RWA [41]. Furthermore, the RWA solution entirely misses intracavity squeezing [36]. Thus, we see that corrections to RWA can lead to qualitatively different features, which is a second major result of our work.

In contrast to unconditional BAE measurements, where CR terms are always detrimental to quantum correlations $[6,42]$, we find that, under continuous monitoring, quantum correlations can be stronger in their presence. Physically, this fact can be traced back to the additional channels opened by CR terms [see Fig. 1(d)]. Indeed, as the backaction spreads more, so do the conditioning effects. The inclusion of CR terms favors a correlated reduction of the uncertainty, which qualitatively accounts for the emergence of entanglement. Remarkably, in the strong-coupling regime we observe the joint presence of conditional optical squeezing, mechanical squeezing, and entanglement. This unusual set of properties has been predicted for the ground state of a pair of bosonic modes in the ultrastrong coupling regime $[43,44]$ and observed in analog quantum simulation of that model [45]. Continuous monitoring could make the same phenomenology accessible without such stringent experimental requirements.

Conditional entanglement in a three-mode optomechanical system.-Now, we consider two mechanical resonators of frequency $\omega_{m, 1}$ and $\omega_{m, 2}$ coupled to a common cavity mode, as sketched in Fig. 1(b). Measuring the output cavity field can induce conditional EPR-like entanglement between them $[13,15,46]$. Following Ref. [13], we introduce the mean and the relative mechanical frequencies $\omega=\left(\omega_{m, 1}+\omega_{m, 2}\right) / 2, \Omega=\left(\omega_{m, 1}-\omega_{m, 2}\right) / 2$ (we assume $\left.\omega_{m, 1}>\omega_{m, 2}\right)$ and the collective EPR mechanical variables

$$
\hat{X}_{ \pm}=\frac{\hat{X}_{m, 1} \pm \hat{X}_{m, 2}}{\sqrt{2}}, \quad \hat{P}_{ \pm}=\frac{\hat{P}_{m, 1} \pm \hat{P}_{m, 2}}{\sqrt{2}},
$$

that satisfy $\left[\hat{X}_{ \pm}, \hat{P}_{ \pm}\right]=i,\left[\hat{X}_{ \pm}, \hat{P}_{\mp}\right]=0$. In terms of $\hat{X}_{+}$and $\hat{P}_{-}$, all-mechanical entanglement is certified by the violation of Duan's inequality $\sigma_{X_{+}}^{2}+\sigma_{P_{-}}^{2} \geq 1$ [47]. Amplitude modulation of a resonant drive at $\omega$ results in the Hamiltonian

$$
\hat{H}_{I}(t)=\Omega\left(\hat{X}_{+} \hat{X}_{-}+\hat{P}_{+} \hat{P}_{-}\right)-\sqrt{2} g \hat{X}_{c} \hat{X}_{+}+\hat{H}_{\mathrm{CR}} .
$$

In the limit $\omega \gg \kappa, \mathrm{CR}$ terms can be dropped and Eq. (9) becomes a perfect two-mode QND interaction $[12,13]$. This is due to the fact that $\hat{H}_{\mathrm{QND}}=\hat{H}_{I}(t)-\hat{H}_{\mathrm{CR}}(t)$ couples $\hat{X}_{+}$ and $\hat{P}_{-}$in the same way as for simple harmonic motion, so that the interaction with the cavity turns into a joint continuous measurement of both $\hat{X}_{+}$and $\hat{P}_{-}$. Since $\hat{X}_{+}$
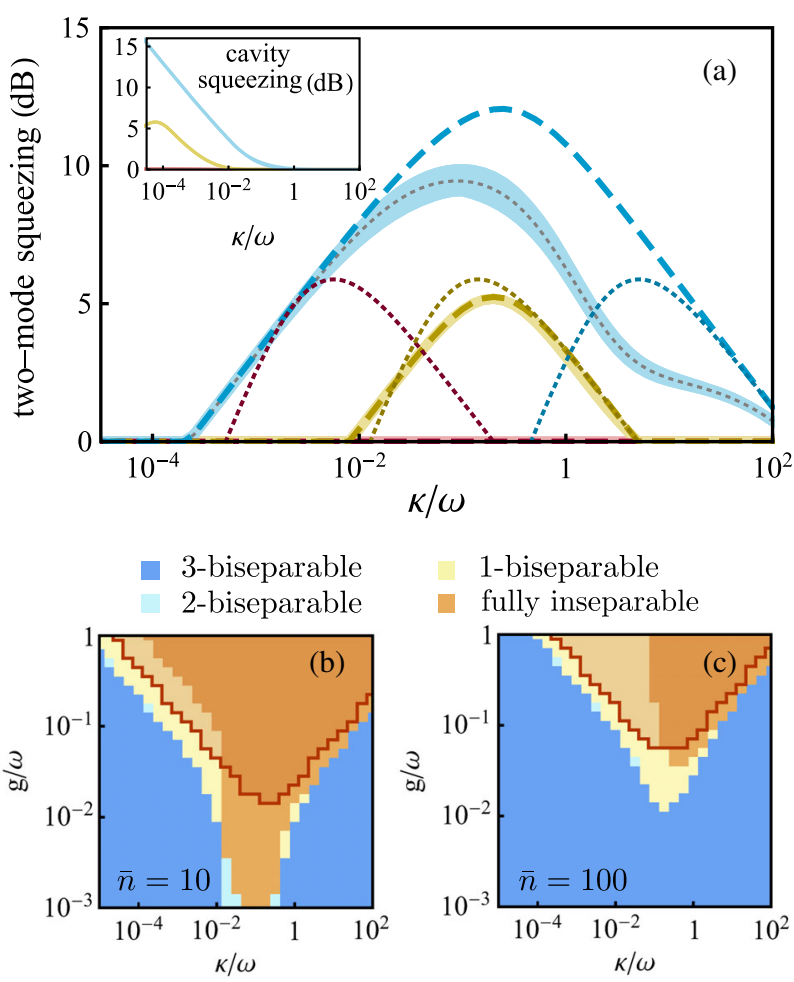

FIG. 4. (a) Mechanical two-mode squeezing (in dB) assuming the RWA (dashed curves), beyond the RWA (lighter shaded areas), and in the adiabatic limit (dotted darker curves). The curves are for $g=0.01 \omega$ (red, which is zero), $g=0.05 \omega$ (yellow), and $g=0.3 \omega$ (cyan). As in Fig. 3, the dotted gray curve shows the average two-mode squeezing (taken over $2 \pi / \omega$ ). In the inset the conditional cavity squeezing is shown. Other parameters are $\Omega=0.1 \omega, \gamma=10^{-4} \omega, \bar{n}=10, \eta=1$. (b) Inseparability structure of the conditional three-mode optomechanical system. The shaded region marks the presence of mechanical two-mode squeezing. Other parameters as in (a). (c) Same as (b) except for $\bar{n}=100$.

and $\hat{P}_{-}$commute, they can be simultaneously squeezed by the measurement, while the backaction is confined to $\hat{P}_{+}$ and $\hat{X}_{-}$[12]. If their combined uncertainties are reduced below twice the zero-point level, the measurement induces conditional mechanical entanglement, in the form of twomode squeezing.

In Fig. 4(a), we quantify two-mode squeezing through the violation of Duan's bound. We observe a trade-off which can be physically understood as in the single-mode case [cf. Fig. 2], although a simple analytic expression [like Eq. (5)] is no longer available. The effects due to CR terms in Eq. (9), responsible for the reduction of the entanglement and the appearance of cavity squeezing for $g>\kappa$, are akin to our findings for the single-mode case [cf. Figs. 3 (a) and 3(c)]. We compare our result with the prediction derived in the adiabatic limit (dotted curves, see Ref. [13] for the expressions), which is only accurate for $\gamma \ll \Omega$, $g \ll \kappa \ll \omega$. Decreasing the coupling, the adiabatic approximation predicts a constant amount of entanglement, only 
shifted towards smaller sideband parameters. This prediction can fail dramatically (see red curve), while our theory correctly quantifies mechanical entanglement in the experimentally relevant good-cavity limit.

Finally, we study the full conditional dynamics of the three-mode optomechanical system, described by Eq. (4), with the appropriate expressions given in the SM [36]. We can determine the separable or entangled nature of the system with respect to all the possible bipartitions, i.e., $\left(\hat{a} \mid \hat{b}_{1} \hat{b}_{2}\right),\left(\hat{b}_{1} \mid \hat{a} \hat{b}_{2}\right)$, and $\left(\hat{b}_{2} \mid \hat{a} \hat{b}_{1}\right)$, leading to the notion of $k$-biseparable states [48]. In particular, there are states that are entangled for any bipartition of the modes [49]; these states are called fully inseparable and possess genuine tripartite entanglement. In Figs. 4(b) and 4(c), we show the inseparability structure induced by the two-mode QND measurement. We find ample regions where genuinely tripartite entanglement and mechanical two-mode squeezing (marked by the shaded area) coexist, which survive even for large thermal occupation. Tripartite entanglement in optomechanical devices has been considered in Refs. [50,51], however, not under continuous monitoring. Most remarkably, our study shows that continuous monitoring can induce nonclassical features at every "layer" of the three-mode system: at the single-mode level, the cavity field is squeezed [cf. inset panel (a)]; the two-mode mechanical state is entangled, and the optomechanical system as a whole displays genuine multipartite entanglement [Figs. 4(b) and 4(c)].

Discussions and conclusions.-Our results are of direct relevance for ultrasensitive measurements and feedbackassisted control [18,21]. Ultralow dissipation optomechanical sensors featuring large cooperativities operate beyond the adiabatic limit. As we showed, in this regime, the enhanced precision achievable (in terms of squeezing) $\sigma_{X_{m} \text {, slow }}^{2} \approx\left(8 \Gamma_{\text {th }}^{3} / \kappa^{2} \Gamma_{\text {meas }}\right)^{1 / 4}$ can be related to the thermal decoherence rate $\Gamma_{\text {th }}=\gamma \bar{n}$ and to the BAE measurement rate $\Gamma_{\text {meas }}=4 \eta g^{2} / \kappa$. This provides a benchmark for the performance of linear position sensors working beyond the SQL and a basic requirement for implementing real-time (Markovian) quantum feedback control. Our results also extend measurement-based control to multiple degrees of freedom. In particular, the possibility of jointly addressing mechanical squeezing, intracavity squeezing, and optomechanical entanglement would be useful for ultrasensitive measurements, e.g., gravitational wave detection [52], quantum information processing [28], as well as fundamental study of quantum decoherence $[53,54]$.

We thank A. Schliesser for discussions at an early stage of the project. M. B. thanks F. Albarelli, M. Genoni, and A. Serafini for useful discussions. D. M. acknowledges support by the Horizon 2020 ERC Advanced Grant Quantum Emitters in non-conventional baths (Grant Agreement No. 742102). A. N. acknowledges a University Research Fellowship from the Royal Society and additional support from the Winton Programme for the Physics of Sustainability. This work was supported by the European Union's Horizon 2020 research and innovation programme under Grant Agreement No. 732894 (FET Proactive HOT).

[1] A. A. Clerk, M. H. Devoret, S. M. Girvin, F. Marquardt, and R. J. Schoelkopf, Rev. Mod. Phys. 82, 1155 (2010).

[2] M. F. Bocko and R. Onofrio, Rev. Mod. Phys. 68, 755 (1996).

[3] V. B. Braginsky, Y. I. Vorontsov, and K. S. Thorne, Science 209, 547 (1980).

[4] K. S. Thorne, R. W. P. Drever, C. M. Caves, M. Zimmermann, and V. D. Sandberg, Phys. Rev. Lett. 40, 667 (1978).

[5] C. M. Caves, K. S. Thorne, R. W. P. Drever, V. D. Sandberg, and M. Zimmermann, Rev. Mod. Phys. 52, 341 (1980).

[6] A. A. Clerk, F. Marquardt, and K. Jacobs, New J. Phys. 10, 095010 (2008).

[7] J. B. Hertzberg, T. Rocheleau, T. Ndukum, M. Savva, A. A. Clerk, and K. C. Schwab, Nat. Phys. 6, 213 (2010).

[8] J. Suh, A. J. Weinstein, C. U. Lei, E. E. Wollman, S. K. Steinke, P. Meystre, A. A. Clerk, and K. C. Schwab, Science 344, 1262 (2014).

[9] I. Shomroni, L. Qiu, D. Malz, A. Nunnenkamp, and T. J. Kippenberg, Nat. Commun. 10, 2086 (2019).

[10] G. Vasilakis, H. Shen, K. Jensen, M. Balabas, D. Salart, B. Chen, and E. S. Polzik, Nat. Phys. 11, 389 (2015).

[11] M. Tsang and C. M. Caves, Phys. Rev. Lett. 105, 123601 (2010).

[12] M. Tsang and C. M. Caves, Phys. Rev. X 2, 031016 (2012).

[13] M. J. Woolley and A. A. Clerk, Phys. Rev. A 87, 063846 (2013).

[14] C. F. Ockeloen-Korppi, E. Damskägg, J.-M. Pirkkalainen, A. A. Clerk, M. J. Woolley, and M. A. Sillanpää, Phys. Rev. Lett. 117, 140401 (2016).

[15] K. Hammerer, M. Aspelmeyer, E. S. Polzik, and P. Zoller, Phys. Rev. Lett. 102, 020501 (2009).

[16] K. Zhang, P. Meystre, and W. Zhang, Phys. Rev. A 88, 043632 (2013).

[17] C. B. Møller, R. A. Thomas, G. Vasilakis, E. Zeuthen, Y. Tsaturyan, M. Balabas, K. Jensen, A. Schliesser, K. Hammerer, and E.S. Polzik, Nature (London) 547, 191 (2017).

[18] D. J. Wilson, V. Sudhir, N. Piro, R. Schilling, A. Ghadimi, and T. J. Kippenberg, Nature (London) 524, 325 (2015).

[19] V. Sudhir, D. J. Wilson, R. Schilling, H. Schütz, S. A. Fedorov, A. H. Ghadimi, A. Nunnenkamp, and T. J. Kippenberg, Phys. Rev. X 7, 011001 (2017).

[20] M. Rossi, N. Kralj, S. Zippilli, R. Natali, A. Borrielli, G. Pandraud, E. Serra, G. Di Giuseppe, and D. Vitali, Phys. Rev. Lett. 119, 123603 (2017).

[21] M. Rossi, D. Mason, J. Chen, Y. Tsaturyan, and A. Schliesser, Nature (London) 563, 53 (2018).

[22] H. M. Wiseman and G. J. Milburn, Phys. Rev. A 47, 642 (1993). 
[23] H. M. Wiseman and G. J. Milburn, Quantum Measurement and Control (Cambridge University Press, Cambridge, England, 2009).

[24] K. Jacobs and D. A. Steck, Contemp. Phys. 47, 279 (2006).

[25] H. Kwon, K. C. Tan, T. Volkoff, and H. Jeong, Phys. Rev. Lett. 122, 040503 (2019).

[26] L. Garbe, S. Felicetti, P. Milman, T. Coudreau, and A. Keller, Phys. Rev. A 99, 043815 (2019).

[27] C. Weedbrook, S. Pirandola, R. García-Patrón, N. J. Cerf, T. C. Ralph, J. H. Shapiro, and S. Lloyd, Rev. Mod. Phys. 84, 621 (2012).

[28] O. Houhou, H. Aissaoui, and A. Ferraro, Phys. Rev. A 92, 063843 (2015).

[29] O. Houhou, D. W. Moore, S. Bose, and A. Ferraro, arXiv:1809.09733.

[30] A. C. Doherty and K. Jacobs, Phys. Rev. A 60, 2700 (1999).

[31] M. Rossi, D. Mason, J. Chen, and A. Schliesser, arXiv: 1812.00928.

[32] S. Hacohen-Gourgy, L. S. Martin, E. Flurin, V. V. Ramasesh, K. B. Whaley, and I. Siddiqi, Nature (London) 538, 491 (2016).

[33] A. Chantasri, M. E. Kimchi-Schwartz, N. Roch, I. Siddiqi, and A. N. Jordan, Phys. Rev. X 6, 041052 (2016).

[34] K. W. Murch, S. J. Weber, C. Macklin, and I. Siddiqi, Nature (London) 502, 211 (2013).

[35] M. Aspelmeyer, T. J. Kippenberg, and F. Marquardt, Rev. Mod. Phys. 86, 1391 (2014).

[36] See Supplemental Material at http://link.aps.org/ supplemental/10.1103/PhysRevLett.123.093602 for details about the conditional dynamics.

[37] M. G. Genoni, L. Lami, and A. Serafini, Contemp. Phys. 57, 331 (2016).

[38] A. Serafini, Quantum Continuous Variables: A Primer of Theoretical Methods (CRC Press, United Kingdom, 2017).
[39] In the fast-cavity limit the homodyne current can be approximated as $I(t) d t \approx 2 \sqrt{\gamma \eta \mathcal{C}}\left\langle\hat{X}_{m}\right\rangle d t+d W_{t}$ which shows that the problem effectively reduces to the continuous measurement of the mechanical amplitude quadrature.

[40] M. G. Genoni, J. Zhang, J. Millen, P. F. Barker, and A. Serafini, New J. Phys. 17, 073019 (2015).

[41] T. Yu and J. H. Eberly, Science 323, 598 (2009).

[42] D. Malz and A. Nunnenkamp, Phys. Rev. A 94, 053820 (2016).

[43] C. Ciuti and I. Carusotto, Phys. Rev. A 74, 033811 (2006).

[44] C. Ciuti and I. Carusotto, Phys. Rev. A 74, 033811 (2006).

[45] D. Marković, S. Jezouin, Q. Ficheux, S. Fedortchenko, S. Felicetti, T. Coudreau, P. Milman, Z. Leghtas, and B. Huard, Phys. Rev. Lett. 121, 040505 (2018).

[46] E. S. Polzik and K. Hammerer, Ann. Phys. (Berlin) 527, A15 (2015).

[47] L.-M. Duan, G. Giedke, J. I. Cirac, and P. Zoller, Phys. Rev. Lett. 84, 2722 (2000).

[48] G. Giedke, B. Kraus, M. Lewenstein, and J. I. Cirac, Phys. Rev. A 64, 052303 (2001).

[49] On the other hand, we recall that three-biseparable states can be either separable or bound entangled states.

[50] C. Genes, A. Mari, P. Tombesi, and D. Vitali, Phys. Rev. A 78, 032316 (2008).

[51] Y.-D. Wang, S. Chesi, and A. A. Clerk, Phys. Rev. A 91, 013807 (2015).

[52] Y. Ma, H. Miao, B. H. Pang, M. Evans, C. Zhao, J. Harms, R. Schnabel, and Y. Chen, Nat. Phys. 13, 776 (2017).

[53] S. Nimmrichter, K. Hornberger, and K. Hammerer, Phys. Rev. Lett. 113, 020405 (2014).

[54] S. McMillen, M. Brunelli, M. Carlesso, A. Bassi, H. Ulbricht, M. G. A. Paris, and M. Paternostro, Phys. Rev. A 95, 012132 (2017). 